Kiran T. Thakur, MD*

John C. Probasco, MD*

Sara E. Hocker, MD

Kelly Roehl, MS, RD

Bobbie Henry, RD

Eric H. Kossoff, MD

Peter W. Kaplan, MB,

FRCP

Romergryko G.

Geocadin, MD

Adam L. Hartman, MD

Arun Venkatesan, MD, PhD*

Mackenzie C. Cervenka, MD*

Correspondence to

Dr. Cervenka:

mcerven1@jhmi.edu

\section{Ketogenic diet for adults in super-refractory status epilepticus}

\section{全}

\section{ABSTRACT}

Objective: To describe a case series of adult patients in the intensive care unit in super-refractory status epilepticus (SRSE; refractory status lasting 24 hours or more despite appropriate anesthetic treatment) who received treatment with the ketogenic diet (KD).

Methods: We performed a retrospective case review at 4 medical centers of adult patients with SRSE treated with the KD. Data collected included demographic features, clinical presentation, diagnosis, EEG data, anticonvulsant treatment, and timing and duration of the KD. Primary outcome measures were resolution of status epilepticus (SE) after initiation of KD and ability to wean from anesthetic agents.

Results: Ten adult patients at 4 medical centers were started on the KD for SRSE. The median age was 33 years (interquartile range [IQR] 21), 4 patients (40\%) were male, and 7 (70\%) had encephalitis. The median duration of SE before initiation of KD was 21.5 days (IQR 28) and the median number of antiepileptic medications used before initiation of KD was 7 (IQR 7). Ninety percent of patients achieved ketosis, and SE ceased in all patients achieving ketosis in a median of 3 days (IQR 8). Three patients had minor complications of the KD including transient acidosis and hypertriglyceridemia and 2 patients ultimately died of causes unrelated to the KD.

Conclusion: We describe treatment of critically ill adult patients with SRSE with the KD, with $90 \%$ of patients achieving resolution of SE. Prospective trials are warranted to examine the efficacy of the KD in adults with refractory SE.

Classification of evidence: This study provides Class IV evidence that for intensive care unit patients with refractory SE, a KD leads to resolution of the SE. Neurology ${ }^{\circledR}$ 2014;82:665-670

\section{GLOSSARY}

AED = antiepileptic drug; GCS = Glasgow Coma Scale; ICU = intensive care unit; IQR = interquartile range; $\mathbf{K D}=$ ketogenic diet; LOS = length of stay; MAD = modified Atkins diet; RSE = refractory status epilepticus; $\mathbf{S E}$ = status epilepticus; SRSE $=$ super-refractory status epilepticus.

Super-refractory status epilepticus (SRSE) is defined as status epilepticus (SE) that continues for at least 24 hours after initiation of general anesthetic medications, including cases in which the SE recurs with reduction or withdrawal of anesthesia. ${ }^{1}$ SRSE holds a high risk of morbidity and mortality and has been associated with conditions such as encephalitis, anoxic brain injury, and intracranial hemorrhage. ${ }^{2}$

The ketogenic diet (KD) and modified Atkins diet (MAD) are high fat, low carbohydrate, adequate protein diets designed to mimic the fasting state that have been proven as effective dietary therapies for some children with epilepsy. ${ }^{3,4}$ Small open-label studies suggest efficacy in adults as well. ${ }^{5}$ Despite their established success in children with refractory epilepsy, only a few case series from single centers describe the use of $\mathrm{KD}$ and MAD in adults with refractory SE (RSE) and SRSE. ${ }^{6-11}$ Given the severity of illness in patients with SRSE, there is a critical need

*These authors contributed equally to this work.

From the Departments of Neurology (K.T.T., J.C.P., E.H.K., R.G.G., A.L.H., A.V., M.C.C.) and Pediatrics (E.H.K., A.L.H.), Johns Hopkins Hospital, Baltimore, MD; Department of Neurology (S.E.H.), Mayo Clinic, Rochester, MN; Department of Food and Nutrition Services (K.R.), Rush University Medical Center, Chicago, IL; Institute for Clinical and Translational Research (B.H.), Johns Hopkins University, Baltimore; Department of Neurology (P.W.K.), Johns Hopkins Bayview Medical Center, Baltimore; and Departments of Anesthesiology-Critical Care Medicine, Neurosurgery, and Medicine (R.G.G.), Johns Hopkins University School of Medicine, Baltimore, MD.

Go to Neurology.org for full disclosures. Funding information and disclosures deemed relevant by the authors, if any, are provided at the end of the article. 


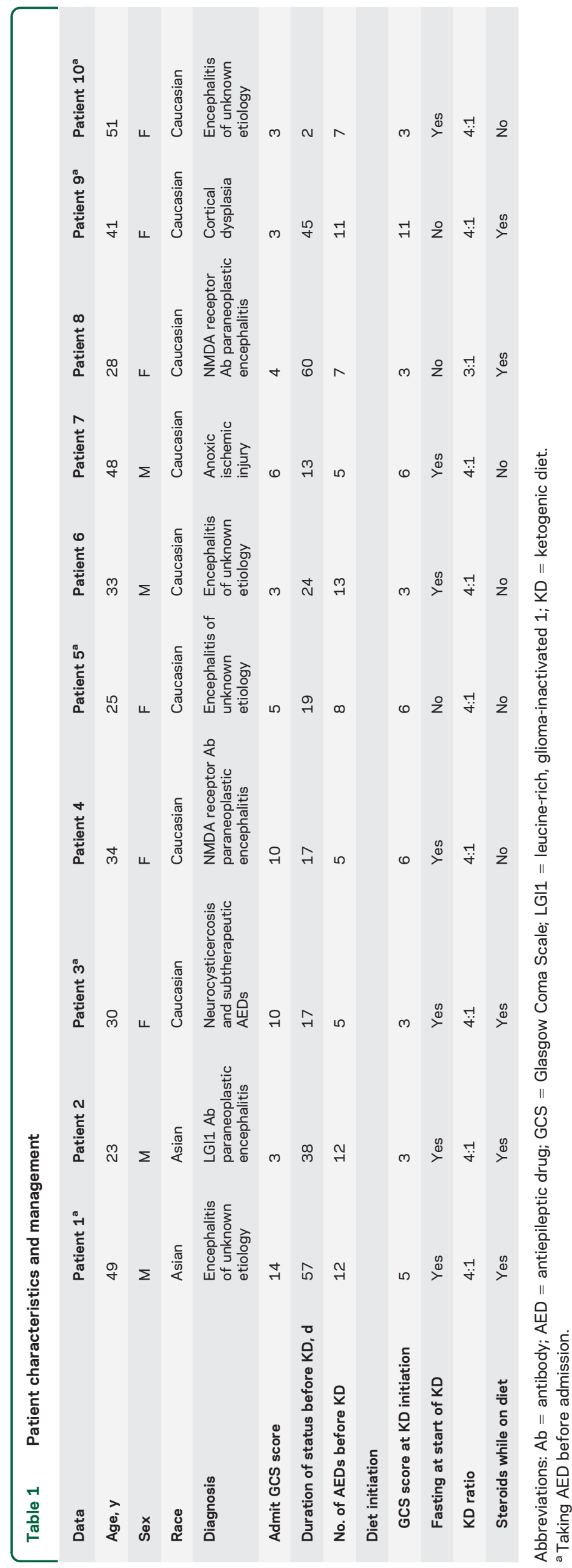

for new therapies to halt ongoing seizure activity. We investigated $\mathrm{KD}$ as a treatment for critically ill adults with SRSE treated in intensive care units (ICUs).

METHODS Standard protocol approvals, registrations, and patient consents. The Johns Hopkins University, Mayo Clinic, Rochester, and Rush University Medical Center Institutional Review Boards approved this study.

Population. A retrospective review of patients older than 17 years in SRSE who were treated with $\mathrm{KD}$ at 4 medical centers between 2010 and 2013 (Johns Hopkins Hospital; Johns Hopkins Bayview Medical Center; Mayo Clinic, Rochester; and Rush University Medical Center) was performed. SRSE was defined as SE (convulsive and/or nonconvulsive) that continued 24 hours or more after the initiation of general anesthetic medications (i.e., propofol, high-dose barbiturates, high-dose benzodiazepines, and ketamine), or recurred after wean or discontinuation of general anesthetics.

Clinical data. Data collected included demographics, clinical diagnosis, history of seizures or SE, total hospital length of stay (LOS), ICU LOS, and Glasgow Coma Scale (GCS) score on admission and at $\mathrm{KD}$ initiation. EEG data collected on $\mathrm{KD}$ initiation, on $\mathrm{KD}$ wean, and at discharge were interpreted by a boardcertified electroencephalographer (M.C.C., P.W.K.). Duration of SE before initiation of diet and number of antiepileptic drugs (AEDs) used before $\mathrm{KD}$ initiation were analyzed. Timing of $\mathrm{KD}$ with respect to seizure onset, $\mathrm{KD}$ duration, presence of serum and urine ketones, and $\mathrm{KD}$ side effects were also collected.

Outcome variables. The ability to wean patients from anesthetic agents within 1 and 2 weeks of $\mathrm{KD}$ initiation and seizure freedom were the primary outcome measures used to demonstrate benefit. Secondary outcome measures included hospital and ICU LOS, EEG features, number of AEDs, GCS and modified Rankin Scale scores at discharge, disposition location (home, rehabilitation, nursing home, death), and modified Rankin Scale score at follow-up. Additional follow-up information gathered included seizure frequency and whether the patient was continued on the $\mathrm{KD}$ or transitioned to the MAD.

Data were confirmed by review of physicians' notes, laboratory results, neuroimaging studies, and other supporting data. Medians and interquartile ranges (IQRs) were calculated for all continuous variables and proportions for all categorical variables. The primary research question was whether $\mathrm{KD}$ led to resolution of SE in patients with SRSE in the ICU (Class IV evidence).

RESULTS Clinical characteristics. Ten adult patients in ICUs at 4 tertiary-care medical centers (2 patients at Johns Hopkins Hospital; 4 at Johns Hopkins Bayview Medical Center; 2 at Mayo Clinic, Rochester; and 2 at Rush University Medical Center) were treated with $\mathrm{KD}$ for SRSE. The median age was 33 years (IQR 21), 4 patients (40\%) were male, and $7(70 \%)$ were Caucasian. Six patients $(60 \%)$ had a history of seizures, with 5 patients $(50 \%)$ taking AEDs before admission (table 1).

Seven patients were diagnosed with encephalitis (3 with antibody-positive paraneoplastic encephalitis and 4 with unknown etiology despite extensive serologic, molecular, and/or histopathologic analysis), one 


\begin{tabular}{|c|c|c|c|c|c|c|c|c|c|c|}
\hline Patient outcomes and fol & & & & & & & & & & \\
\hline Data & Patient 1 & Patient 2 & Patient 3 & Patient 4 & Patient 5 & Patient 6 & Patient 7 & Patient 8 & Patient 9 & Patient 10 \\
\hline \multicolumn{11}{|l|}{ Outcomes } \\
\hline Ketosis achieved & Yes & Yes & Yes & Yes & Yes & Yes & Yes & No & Yes & Yes \\
\hline Time to ketosis, ${ }^{a} \mathrm{~d}$ & 1 & 7 & 1 (ketonuria) & 3 & 2 & 7 (ketonuria) & 4 & NA & 3 & 6 \\
\hline Total length of KD, $d$ & 24 & 41 & 19 & 16 & 4 & 23 & 4 & 13 & 30 & 9 \\
\hline Side effects ${ }^{b}$ & $\uparrow T \mathrm{TG}$ & Acidosis & None & None & $\uparrow T \mathrm{TG}$ & None & None & None & None & None \\
\hline Days to wean anesthetics after diet & 3 & 6 & 5 & 5 & 0 & 5 & 4 & NA & 0 & 6 \\
\hline Total days on anesthetic agents & 34 & 64 & 27 & 27 & 8 & 10 & 10 & 76 & 29 & 9 \\
\hline Time to EEG seizure resolution, $d$ & 1 & 31 & 12 & 2 & 3 & 5 & 7 & NA & 3 & 1 \\
\hline ICU length of stay, $d$ & 43 & 41 & 30 & 38 & 25 & 59 & 28 & 76 & 54 & 10 \\
\hline Total length of hospitalization, $d$ & 94 & 142 & 37 & 81 & 37 & 86 & 28 & 97 & 57 & 16 \\
\hline $\begin{array}{l}\text { Time to initiation of solid food after KD } \\
\text { initiation, } d\end{array}$ & 24 & 97 & 15 & 24 & 11 & 34 & NA & NA & 30 & 9 \\
\hline No. of AEDs at hospital discharge & 4 & 4 & 3 & 2 & 4 & 6 & 3 & NA & 5 & 6 \\
\hline Discharge GCS score & 14 & 14 & 14 & 12 & 14 & 11 & 8 & 3 & 11 & 15 \\
\hline mRS score at discharge & 2 & 4 & 1 & 3 & 3 & 5 & 5 & 6 & 5 & 4 \\
\hline Disposition & $\begin{array}{l}\text { Rehabilitation } \\
\text { facility }\end{array}$ & $\begin{array}{l}\text { Rehabilitation } \\
\text { facility }\end{array}$ & $\begin{array}{l}\text { Rehabilitation } \\
\text { facility }\end{array}$ & $\begin{array}{l}\text { Rehabilitation } \\
\text { facility }\end{array}$ & $\begin{array}{l}\text { Rehabilitation } \\
\text { facility }\end{array}$ & $\begin{array}{l}\text { Rehabilitation } \\
\text { facility }\end{array}$ & $\begin{array}{l}\text { Nursing home/ } \\
\text { skilled nursing } \\
\text { facility }\end{array}$ & Death & $\begin{array}{l}\text { Respiratory care } \\
\text { unit/ventilatory } \\
\text { wean unit }\end{array}$ & $\begin{array}{l}\text { Rehabilitation } \\
\text { facility }\end{array}$ \\
\hline \multicolumn{11}{|l|}{ Follow-up } \\
\hline $\mathrm{mRS}$ score at $6 \mathrm{mo}$ & 1 & $<6 \mathrm{mo}, 4$ & Unknown & $<6 \mathrm{mo}, 3$ & 3 & 0 & 6 & 6 & 3 & 2 \\
\hline Transition to MAD & Yes & No & Yes & No & Yes & No & No & No & Yes & Yes \\
\hline Seizure freedom at 6-mo follow-up & Yes & $<6$ mo, yes & Unknown & $<6 \mathrm{mo}$, yes & $\begin{array}{l}\text { No (20 FDS, } \\
1 \mathrm{GTC} / \mathrm{mo})\end{array}$ & $\begin{array}{l}\text { No }(2 \text { FDS in } \\
6 \mathrm{mo})\end{array}$ & Deceased & Deceased & $\begin{array}{l}\text { No (6 FDS in } 6 \\
\text { mo) }\end{array}$ & $\begin{array}{l}\text { No ( } \downarrow \\
\text { admissions } \\
\text { for status) }\end{array}$ \\
\hline
\end{tabular}

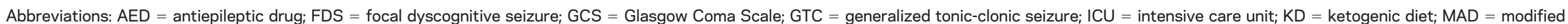
Atkins diet; $\mathrm{mRS}$ = modified Rankin Scale; NA = not applicable (because patient did not achieve ketosis or died); $\mathrm{TG}=$ triglycerides.

${ }^{a}$ Ketosis is defined by ketonemia unless indicated.

b Side effects defined as gastrointestinal symptoms, acidosis, hypotension, hyperlipidemia/hypertriglyceridemia ( $\uparrow \mathrm{TG})$, nephrolithiasis, pancreatitis, and cardiomyopathy. 
Table 3 Considerations for implementing ketogenic diet

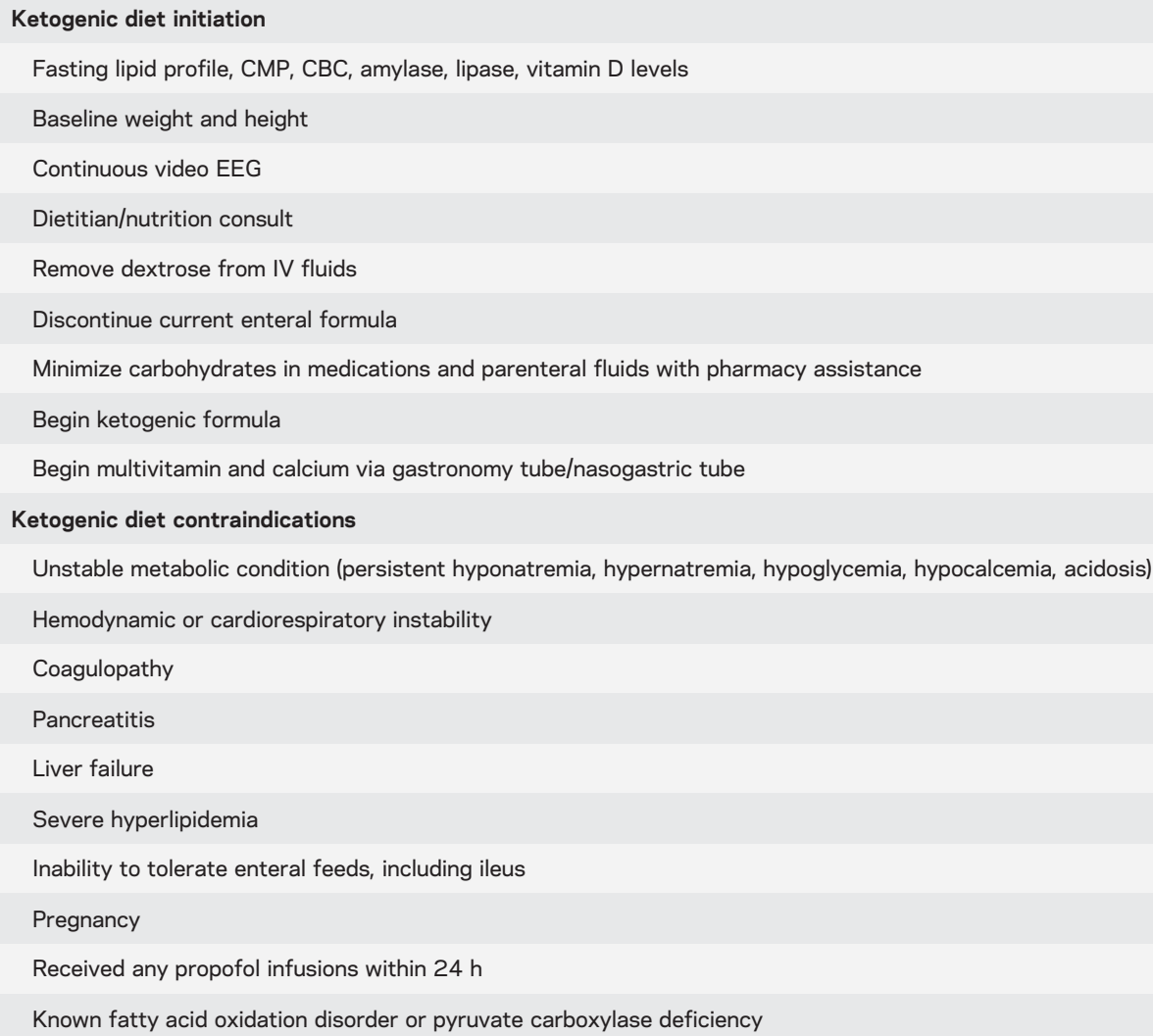

Abbreviations: $\mathrm{CBC}=$ complete blood count; $\mathrm{CMP}=$ comprehensive metabolic panel.

with neurocysticercosis and subtherapeutic AED levels, one with anoxic brain injury, and one with cortical dysplasia (table 1). Five patients were taking AEDs before admission for SRSE because of known diagnoses of epilepsy. Median admission GCS score was 4.5 (IQR 8), with 7 patients having a GCS score $<8$. Median SE duration before KD initiation was 21.5 days (IQR 28). Median number of AEDs used before $\mathrm{KD}$ initiation was 7 (IQR 7). All patients were in convulsive and/or nonconvulsive SE before $\mathrm{KD}$ initiation. Two of the 3 patients with antibody-mediated encephalitis received at least 2 courses of IV immunoglobulin and/or plasmapheresis before initiation of the $\mathrm{KD}$.

Diet initiation. Nine patients were placed on a 4:1 KD ratio (fat to carbohydrates and protein grams) and one on a 3:1 ratio. At the time of $\mathrm{KD}$ initiation, EEGs showed pharmacologic burst suppression in 2 patients (after at least one prior failed attempt to wean anesthetics), electrographic seizures in 6 patients, slowing with interictal epileptiform activity in one patient, and generalized periodic epileptiform discharges in one patient. Nine patients had a GCS score $<8$ at initiation. Ketosis was achieved in 9 patients within a median of 3 days (IQR 5). Two patients developed hypertriglyceridemia and one had transient acidosis that resolved without interrupting dietary treatment.
The median length of time on the $\mathrm{KD}$ was 17.5 days (IQR 15) (tables 1 and 2).

Outcome after KD initiation. Nine patients (90\%) had resolution of SE within a median of 3 days (IQR 8) after KD initiation. Seven patients had clinical and/ or electrographic seizure resolution within 1 week of diet initiation, and 9 within 1 month. The median days on anesthetic agents after $\mathrm{KD}$ initiation was 5 (IQR 4). On discharge, 6 patients had a GCS score $\geq 12$ (including 4 with known epilepsy taking AEDs before admission). The median number of AEDs prescribed at discharge was 4 (IQR 2.5).

Two patients ultimately died; one died after cardiac arrest in the setting of persistent seizures and exhaustion of therapeutic options, and the family of a second patient withdrew care because of persistent coma, despite seizure control. Five patients transitioned to MAD (table 2). Of these, 2 continue to have intermittent seizures on MAD with decreased frequency compared with baseline. One patient has been seizure-free and was weaned off MAD after 6 months, without recurrence. One patient stopped MAD and continues to have intermittent seizures with AED adjustments. One was lost to follow-up. Three patients have been off $\mathrm{KD}$ since discharge ( 2 are seizure-free, and one has intermittent seizures). 
DISCUSSION We describe 10 adult patients who were treated with KD for SRSE and found that a majority of these patients $(90 \%)$ had resolution of SE within days after diet initiation. Although we describe a small cohort of patients, our study suggests that dietary therapy may have a role in treating adult patients with SRSE.

The use of KD to control SRSE has been previously reported in case reports and small case series. $^{6-11}$ One case in the present series was previously reported in the literature. ${ }^{7}$ Wusthoff et al. ${ }^{10}$ reported the largest number of adult patients (2) treated with KD for RSE to date. These cases and our current findings suggest that $\mathrm{KD}$ is a safe and feasible treatment for critically ill adult patients with RSE and SRSE when implemented appropriately (table 3). An ongoing prospective multicenter trial using a standardized KD protocol will provide further data on the safety and efficacy of KD in critically ill patients with RSE and SRSE.

A major limitation of the current study is its retrospective nature. Many patients received adjunctive treatment with multiple AEDs, surgical intervention,

\section{Comment:}

\section{Should we induce ketosis in super-refractory status epilepticus?}

Persistent or re-emergent status epilepticus occurs in as many as $20 \%$ of patients treated with conventional pharmacotherapy for refractory status epilepticus. ${ }^{1}$ The prognosis associated with this "malignant" or "super-refractory" status epilepticus (SRSE) is not universally poor, making therapeutic efforts worthwhile in some cases. Unfortunately, the literature is dominated by anecdotal reports of last-resort therapies that offer little evidence to guide treatment. ${ }^{2}$

The article in this issue ${ }^{3}$ adds additional retrospective data from 10 patients suggesting that the ketogenic diet may be an effective therapy for some patients in SRSE. Thakur et al. describe resolution of status epilepticus in the 9 patients who achieved ketosis. The addition of these cases to the scattered reports in the literature is intriguing, but is there sufficient evidence to recommend the ketogenic diet as an established treatment option? Certainly not. Is there sufficient evidence to justify the authors' assertion that prospective studies are warranted? I think so, but designing and conducting such a study will be challenging.

In the meantime, should we consider the ketogenic diet as a therapeutic option for patients in SRSE? Perhaps, but not before treatments for which there are more robust data. More case reports do not indicate more efficacy. As an unproven intervention that could be proven ineffective in a prospective study, the ketogenic diet should remain in the same category as hypothermia, emergency surgery, immunologic therapy, and any other option for which the data are incomplete.

1. Holtkamp M, Othman J, Buchheim $\mathrm{K}$, et al. A "malignant" variant of status epilepticus. Arch Neurol 2005;62:1428-1431.

2. Ferlisi M, Shorvon S. The outcome of therapies in refractory and super-refractory convulsive status epilepticus and recommendations for therapy. Brain 2012;135: 2314-2328.

3. Thakur KT, Probasco JC, Hocker SE, et al. Ketogenic diet for adults in super-refractory status epilepticus. Neurology 2014;82:665-670.

Michael Gruenthal, MD, PhD

From the Department of Neurology, Albany Medical College, Albany, NY.

Study funding: No targeted funding reported.

Disclosure: M. Gruenthal serves as a consultant to the New York State Department of Health, receives research support from Eisai Inc. and Upsher-Smith Laboratories Inc., and receives royalty payments from Apple, Inc. for computer software. Go to Neurology.org for full disclosures.
IV steroids, plasmapheresis, and IV immunoglobulin while on the KD. For patients with antibodymediated encephalitis, we were unable to gather detailed information regarding the duration and timing of immune-mediated therapies in relation to the initiation of the KD. While we recorded AEDs used through each patient's clinical course, the specific order and duration given and serum levels were not obtained. We therefore cannot exclude the possibility that other interventions led to clinical improvement and seizure freedom. Also, protocols for KD initiation and monitoring varied. We lacked detailed follow-up information on one patient. Finally, 5 patients discontinued dietary treatment, preventing comment on long-term efficacy of continued dietary therapy in these individuals.

Our case series suggests that the KD is safe and feasible in ICU patients and may have a role in refractory cases. It remains to be shown whether early intervention with $\mathrm{KD}$ and transition to the MAD improves morbidity and mortality in this critically ill population.

\section{AUTHOR CONTRIBUTIONS}

Kiran Thakur, MD, and John Probasco, MD: design, data collection, analysis, and writing of the manuscript. Sara Hocker, MD: design, data collection, and writing of the manuscript. Kelly Roehl, MS, RD: data collection and writing of the manuscript. Bobbie Henry, RD, and Eric Kossoff, MD: design, data analysis, and review of the manuscript. Peter Kaplan, MB, FRCP: design, analysis, writing and review of the manuscript. Romergryko Geocadin, MD, and Adam Hartman, MD: design, analysis, and writing of the manuscript. Arun Venkatesan, MD, PhD: design, analysis, writing and review of the manuscript. Mackenzie Cervenka, MD: design, data collection, analysis, writing and review of the manuscript.

\section{STUDY FUNDING}

No targeted funding reported.

\section{DISCLOSURE}

K. Thakur, J. Probasco, S. Hocker, and K. Roehl report no disclosures. B. Henry: grants from Johns Hopkins Institute for Clinical and Translational Research, funded in part by NIH grants (UL1 TR 000424-06 NCATS), NIH Roadmap for Medical Research, and Nutricia. E. Kossoff: grant from Nutricia; consulting to Atkins Nutritionals, Inc. P. Kaplan: data safety monitoring board for UCB lacosamide TRENdS trial-Duke University. A. Hartman: research support from NIH (NINDS) and Johns Hopkins University School of Medicine; receives income from his clinical practice and reading EEGs (21\% effort); and has provided expert opinion in medicolegal cases. R. Geocadin: NIH grants (supported in part by 5R01HL071568 and RO1 NS074425). A. Venkatesan: grants from NIH, HHMI, Maryland Stem Cell Research Foundation, National Multiple Sclerosis Society; data-safety monitoring for Bristol-Myers Squibb and GlaxoSmithKline. M. Cervenka: grants from Johns Hopkins University School of Medicine Clinician Scientist Award, Nutricia, and NIH (NINDS R01NS075020). Go to Neurology.org for full disclosures.

Received August 22, 2013. Accepted in final form October 22, 2013.

\section{REFERENCES}

1. Shorvon S, Ferlisi M. The outcome of therapies in refractory and super-refractory convulsive status epilepticus and recommendations for therapy. Brain 2012;135:23142328. 
2. Hocker SE, Britton JW, Mandrekar JN, Wijdicks EF, Rabinstein AA. Predictors of outcome in refractory status epilepticus. JAMA 2013;70:72-77.

3. Wilder RM. The effects of ketonemia on the course of epilepsy. Mayo Clin Proc 1921;2:307-308.

4. Kossoff EH, McGrogan JR, Bluml RM, Pillas DJ, Rubenstein JE, Vining EP. A modified Atkins diet is effective for the treatment of intractable pediatric epilepsy. Epilepsia 2006;47:421-424.

5. Payne NE, Cross JH, Sander JW, Sisodiya SM. The ketogenic and related diets in adolescents and adults: a review. Epilepsia 2011;52:1941-1948.

6. Bodenant M, Moreau C, Sejourne $\mathrm{C}$, et al. Interest of the ketogenic diet in a refractory status epilepticus in adults. Rev Neurol 2008;164:194-199.

7. Cervenka MC, Hartman AL, Venkatesan A, Geocadin RG, Kossoff EH. The ketogenic diet for medi- cally and surgically refractory status epilepticus in the neurocritical care unit. Neurocrit Care 2011;15:519-524.

8. Nabbout R, Mazzuca M, Hubert P, et al. Efficacy of ketogenic diet in severe refractory status epilepticus initiating fever induced refractory epileptic encephalopathy in school age children (FIRES). Epilepsia 2010;51:2033-2037.

9. Nam SH, Lee BL, Lee CG, et al. The role of ketogenic diet in the treatment of refractory status epilepticus. Epilepsia 2011;52:e181-e184.

10. Wusthoff CJ, Kranick SM, Morley JF, Christina Bergqvist AG. The ketogenic diet in treatment of two adults with prolonged nonconvulsive status epilepticus. Epilepsia 2010;51:1083-1085.

11. Strzelczyk A, Reif PS, Bauer S, et al. Intravenous initiation and maintenance of ketogenic diet: proof of concept in super-refractory status epilepticus. Seizure 2013;22: 581-583.

\section{Call for Nominations: Editor-in-Chief of Neurology Now}

The AAN seeks self-nominations or nominations of other AAN members for editor-in-chief of Neurology Now ${ }^{\circledR}$. The Academy's bimonthly magazine for patients and caregivers reaches 1.6 million readers per issue. The editor-in-chief serves as the leader and public champion of the magazine and is responsible for setting the editorial vision and direction for the publication during a time of transition from print to digital publishing while continuing the strong tradition of patient-centered education, medical integrity, and high editorial quality that has been established in Neurology Now.

The initial appointment is three years beginning January 1, 2015, with a four-month transition with the current editor-in-chief beginning September 1, 2014. The deadline for nominations is February 28, 2014. A position description, including requirements, is available at AAN.com/view/NNEditor-in-Chief.

\section{Enjoy Big Savings on NEW 2014 AAN Practice Management Webinars Subscriptions}

The American Academy of Neurology offers 14 cost-effective Practice Management Webinars you can attend live or listen to recordings posted online. AAN members can purchase one webinar for $\$ 149$ or subscribe to the entire series for only $\$ 199$. This is new pricing for 2014 and significantly less than 2013 - and big savings from the new 2014 nonmember price of $\$ 199$ per webinar or $\$ 649$ for the subscription. Register today for these and other 2014 webinars at AAN.com/view/pmw14:

January 21 - Correct Coding for Chemodenervation

February 4 - Coding for Neurodiagnostic Procedures Made Easy

March 11 - Bundled Payments: The Role for Neurologists in New Health Care Models 